\title{
Estimating 1-MCP application for Kampuchea guava with data mining technology
}

\begin{abstract}
The application of 1-methylcyclopropene (1-MCP) influences the fruit qualities and may affect fruit sales since customers have different preferences on fruit qualities. However, estimating 1-MCP application requires the lab procedure that consumes time and cost. In this preliminary study, data mining (DM) technology was utilized to achieve fast estimation of 1MCP application based on different qualities of ' Kampuchea' Guava. Five DM algorithms were involved, namely, (i) C4.5, (ii) Library for Support Vector Machine (LibSVM), (iii) Multilayer Perceptron (MLP), (iv) Naive Bayes Classifier (NBC), and (v) Random Forest (RF) to build classification models that understand the behaviour of the past laboratory data. The classification models can then be used for estimating the 1-MCP application fast whenever there are new data available. The result showed that fast estimation can be achieved effectively using LibSVMas which outperformed the others by attaining the lowest errors in four statistical measures.
\end{abstract}

Keyword: Library for Support Vector Machine; Multilayer perceptron; Naive bayes classifier; Random forest 